15

\title{
Влияние продолжительности контакта и глубины индентирования на адгезионную прочность: эксперимент и численное моделирование
}

\author{
() Я.А. Ляшенко, ${ }^{1,2}$ В.Л. Попов ${ }^{1,3,4}$ \\ ${ }^{1}$ Берлинский технический университет, \\ 10623 Берлин, Германия \\ ${ }^{2}$ Сумский государственный университет, \\ 40007 Сумы, Украина \\ ${ }^{3}$ Национальный исследовательский Томский государственный университет, \\ 634050 Томск, Россия \\ ${ }^{4}$ Институт физики прочности и материаловедения, \\ 634055 Томск, Россия \\ e-mail: i.liashenko@tu-berlin.de
}

Поступило в Редакцию 18 февраля 2020 г.

В окончательной редакции 22 марта 2020 г.

Принято к публикации 22 марта 2020 г.

\begin{abstract}
Экспериментально исследован адгезионный контакт между стальным сферическим индентором и фрагментом листа прозрачной мягкой резины, закрепленным на стеклянной подложке. Проведено сравнение полученных экспериментальных данных с результатами аналитической теории и численным моделированием, продемонстрировавшее количественное согласие между этими тремя подходами. Также изучается влияние продолжительности нахождения индентора в контакте и глубины индентирования на значение его адгезионной прочности. Обсуждены особенности экспериментов, проводимых в условиях контролируемого смещения и контролируемой силы.
\end{abstract}

Ключевые слова: адгезия, нормальный контакт, поверхностная энергия, адгезионная прочность, индентирование, эластомеры.

DOI: $10.21883 / J T F .2020 .10 .49812 .57-20$

\section{Введение}

Под термином „адгезия“ традиционно понимается процесс сцепления поверхностей разнородных тел за счет сил межмолекулярного взаимодействия. Адгезионное взаимодействие обеспечивает необходимую механическую связь между клетками и клеточными структурами в биологических организмах, прочность клеевых и спаянных соединений, а также прочность многих промышленных механических конструкций [1-4]. За счет адгезии многие биологические виды получили способность перемещаться по вертикальным поверхностям. Например, для такого перемещения адгезию используют гекконы и многие виды земноводных (древесные квакши, филломедузы). В настоящее время в науке и промышленности существует направление, в рамках которого создают искусственные адгезионные поверхности $[5,6]$, используемые, к примеру, для перемещения деталей в различных технологических процессах. Такие поверхности, как правило, состоят из прикрепленных к плоскости упругого материала равноудаленных цилиндров с основанием различной формы. При этом адгезионная прочность контакта зависит от формы основания цилиндров и их удаленности друг от друга. На основе таких искусственных материалов создают разнообразные механизмы, способные перемещаться по вертикальным поверхностям. Эти механизмы уже используют для манипулирования объектами на роботизированном производ- стве, в перспективе их планируют также применять на космических станциях в условиях открытого космоса [7]. В связи с повышенной актуальностью в последнее время активно проводятся теоретические и экспериментальные исследования, посвященные изучению адгезионного контакта [8-10]. В этих работах было показано, что реальная картина адгезионного взаимодействия выходит далеко за рамки классических теорий типа JKR [11]. Различие с теорией вызвано многими факторами, например, тем, что реальные поверхности всегда имеют шероховатости, а поверхностная энергия неоднородно распределена по поверхности контактирующих тел и изменяется со временем ввиду протекания в зоне контакта химических реакций. Существенное влияние на адгезионные свойства также оказывает загрязнение поверхностей и их износ во время контакта. Причем, как правило, все эти факторы изменяются случайным образом, поэтому для выяснения их влияния необходимо проводить реальный эксперимент, а потом уже создавать подходящую под тот или иной случай модель либо теорию. В настоящей работе предложен именно такой подход. Сперва выполнен цикл экспериментальных исследований, в которых изучается влияние на адгезионную прочность глубины индентирования и времени нахождения индентора в контакте. После чего там, где это возможно, проводится сравнение полученных в работе экспериментальных данных с результатами известных теорий и численного моделирования. 


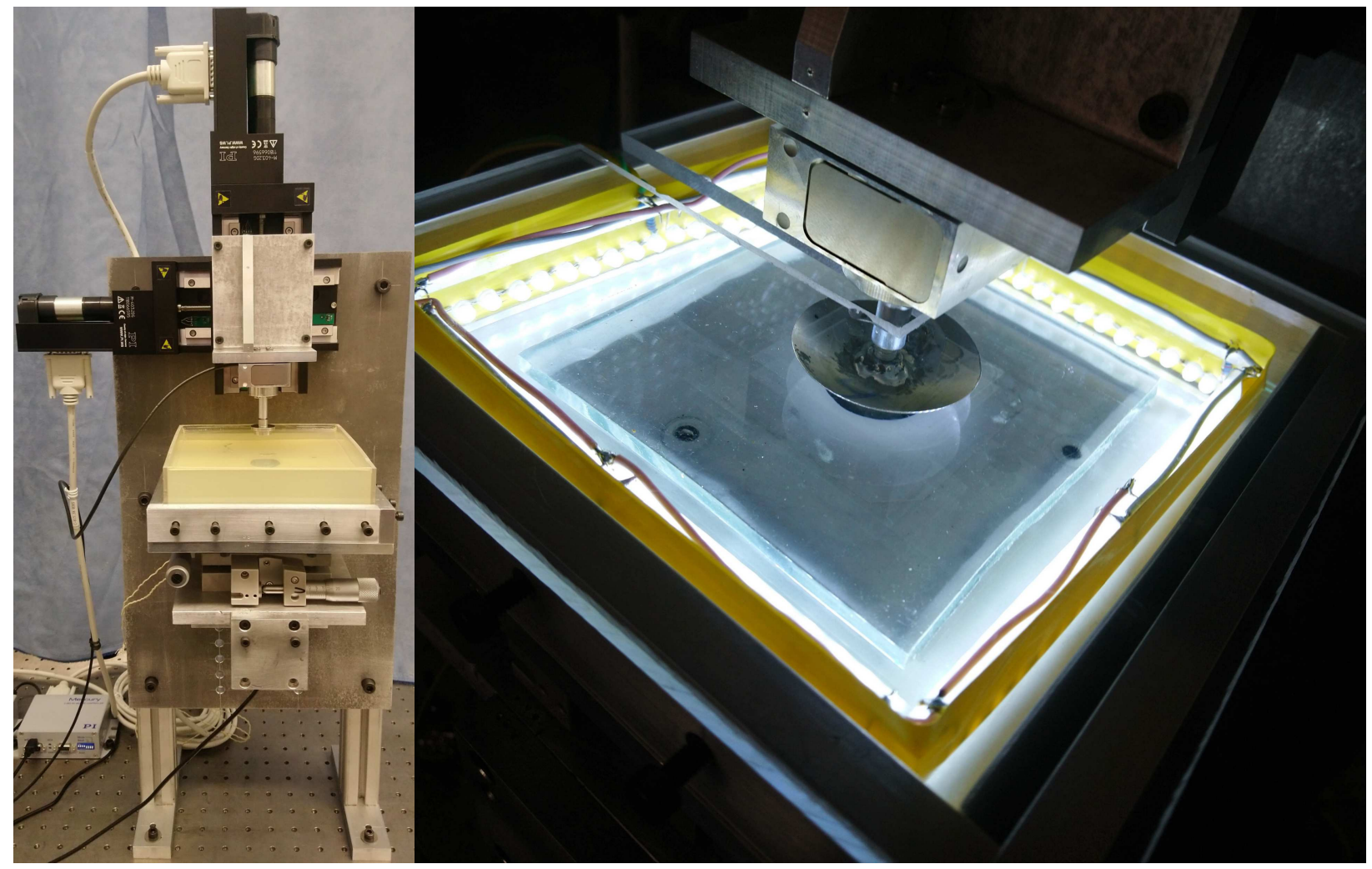

Рис. 1. Внешний вид экспериментальной установки (слева); фотография области контакта со всесторонним светодиодным освещением (справа).

\section{1. Устройство экспериментальной установки и принцип ее работы}

На рис. 1 приведена фотография разработанного нами устройства, которое позволяет изучать адгезионные процессы при произвольной геометрии контакта. В левой части рисунка показан внешний вид установки, а в правой - область контакта между стальным индентором и фрагментом прозрачного резинового листа. Установка позволяет производить нагружение контакта одновременно в нормальном и тангенциальном направлениях по любой заданной траектории. Индентор смещается с помощью соединенных вместе приводов направленного движения M-403.2DG фирмы PI, которые управляются контроллерами C-863 той же фирмы. Эти приводы позволяют производить смещение максимум до $50 \mathrm{~mm}$ с минимальным шагом $0.2 \mu \mathrm{m}$. Однако при изменении направления движения используемые приводы обладают люфтом порядка $10 \mu \mathrm{m}$ что не позволяет точно исследовать гистерезисные явления в адгезионном контакте при малых реверсивных смещениях. Для измерения действующих контактных сил использовался трехосевой сенсор силы K3D40 фирмы ME-Meßsysteme, работающий в диапазоне \pm 10 N. К указанному сенсору и прикреплен индентор, показанный на правой панели на рис. 1. Для усиления получаемого от сенсора электрического сигнала применялся четырехканальный усилитель GSV-1A4
SubD37/2, а для сопряжения усиленного сигнала с компьютером - 16-bit АЦП NI USB-6211. На протяжении индентирования осуществлялось наблюдение за эволюцией контактной площади с помощью видеокамеры Conrad USB разрешением $1600 \times 1200$ pixel. Все фиксирующие элементы установки выполнены из алюминия. Материалы индентора и эластомера выбирались исходя из поставленной задачи. Согласованное управление всеми механизмами устройства осуществляется с помощью компьютерной программы, разработанной в среде LabVIEW. Tраектория движения индентора задается как две (при необходимости кусочные) функции времени, отдельно для нормального и тангенциального направлений. Во время такого движения с фиксированным временным интервалом (обычно один раз в секунду) записываются значения всех трех компонент силы и сохраняются фотографии контактной области.

\section{2. Гистерезис силы при прямом и обратном циклах индентирования и сравнение с теорией}

Нами был проведен эксперимент по индентированию стального сферического индентора, который имел радиус $R=33 \mathrm{~mm}$, в резиновый образец TARNAC CRG N3005 толщиной $h=25 \mathrm{~mm}$, который распола- 

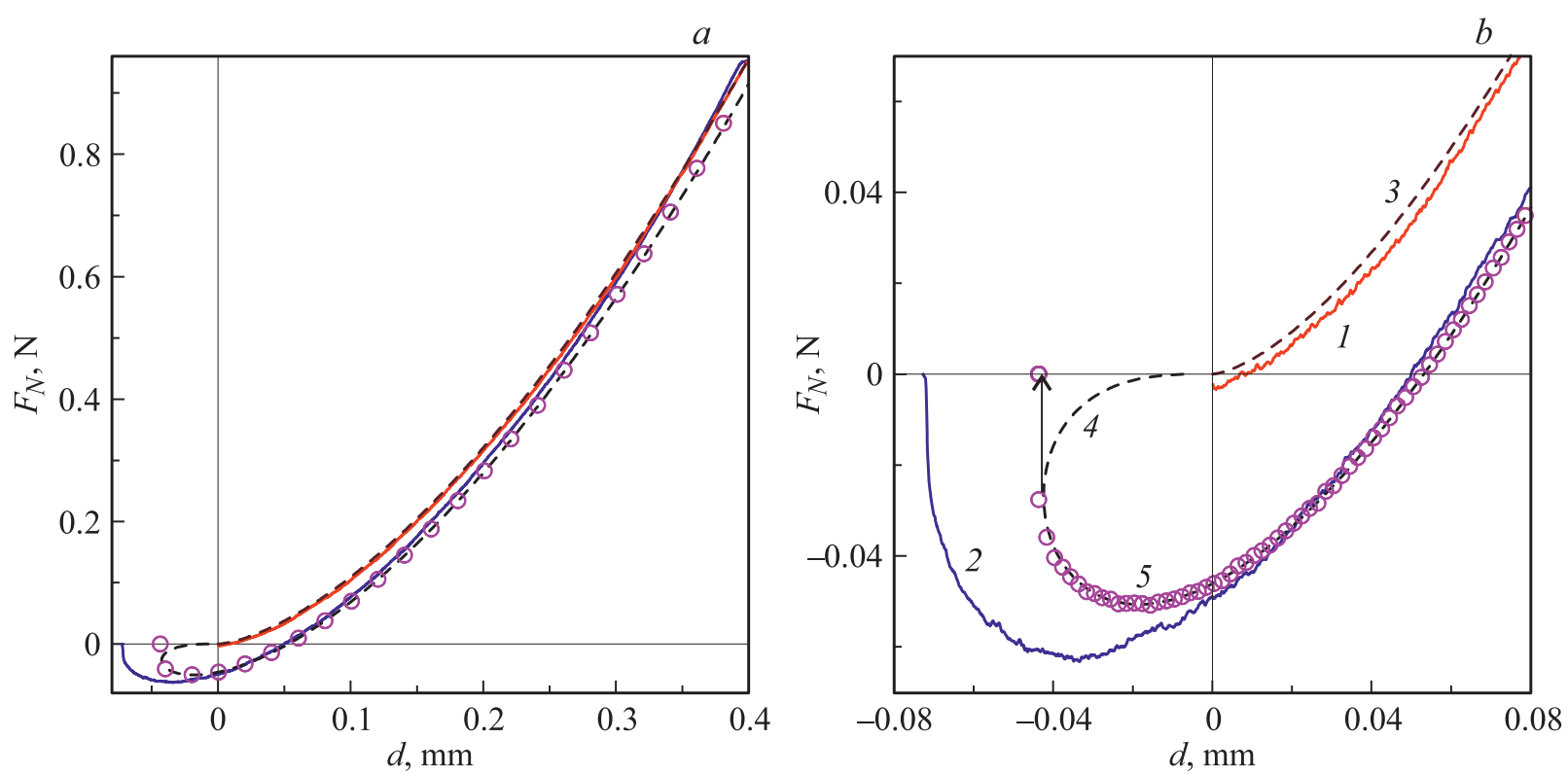

Рис. 2. Зависимости нормальной силы $F_{N}$ от глубины индентирования $d$ при индентировании сферического индентора, имеющего радиус $R=33 \mathrm{~mm}$, в резиновый образец TARNAC CRG N3005 толщиной $h=25 \mathrm{~mm}$. На частях $a$ и $b$ показаны одни и те же кривые: 1 - прямой цикл индентирования (эксперимент); 2 - обратный цикл индентирования (эксперимент); 3 - индентирование без адгезии (теоретический расчет); 4 - индентирование с адгезией (теоретический расчет); 5 - индентирование с адгезией (моделирование методом граничных элементов).

гался на жесткой стеклянной подложке. Этот образец представлял 5 сложенных вместе резиновых листов толщиной $5 \mathrm{~mm}$ каждый, которые прочно прилипают друг к другу при контакте за счет адгезии. В эксперименте осуществлялось индентирование в резиновый образец на глубину $d=0.4 \mathrm{~mm}$, после чего индентор сразу сдвигался в обратном направлении до полного исчезновения контакта. Скорость движения в этих двух фазах индентирования оставалась постоянной и составляла $v=0.1 \mu \mathrm{m} / \mathrm{s}$. Такая скорость была выбрана для исключения влияния вязкоупругих эффектов, изучение которых для аналогичной системы уже проводилось ранее [12]. На рис. 2 показаны зависимости нормальной силы $F_{N}$ от глубины индентирования $d$, измеренные в эксперименте. При этом на рис. 2, $a$ полностью показан диапазон глубин индентирования, а на рис. $2, b$ подробно выделена область, в которой происходит разрушение адгезионного контакта. В остальном части рисунка идентичны - на них показаны одни и те же кривые (всего по 5 зависимостей на каждом рисунке). Здесь сплошной кривой 1 изображена экспериментально измеренная зависимость для фазы индентирования, сплошная кривая $2-$ это экспериментальная зависимость в фазе отрыва. Согласно общепринятой теории JKR [11], в точке первого касания адгезионный контакт должен распространиться до равновесного значения, которое задается балансом между упругой энергией и работой адгезионных сил. При последующем движении индентора в обоих направлениях должна реализоваться единственная зависимость $F_{N}(d)$, определяемая вышеупомянутым балансом энергий, если мы не доходим до критической глубины индентирования в области $d<0$, при которой адгезионный контакт разрушается. Однако проведенный эксперимент однозначно показывает, что процессы индентирования и отрыва описываются различными зависимостями вышеупомянутыми кривыми 1 и 2 . При этом в точке первого касания контакт распространяется лишь незначительно, что обеспечивает довольно низкое абсолютное значение отрицательной нормальной силы $F_{N}$ в начале процесса индентирования. Более того, по мере увеличения глубины индентирования $d$ система быстро выходит в диапазон положительных значений нормальной силы, что также говорит об очень слабом влиянии адгезионного взаимодействия в первоначальной фазе индентирования. Однако этого нельзя сказать о фазе отрыва (экспериментальная кривая 2), в которой измеренная зависимость $F_{N}(d)$ качественно напоминает результаты теории JKR.

При прямом и обратном циклах индентирования помимо зависимости $F_{N}(d)$ гистерезисное поведение показывает также и зависимость площади контакта от глубины вдавливания. На рис. 3 приведены фотографии контактной области для одного и того же значения глубины индентирования $d=0.2 \mathrm{~mm}$ для прямого (кривая 1 на рис. 2, части 1 и 2 на рис. 3) и обратного (кривая 2 на рис. 2, части 3 и 4 на рис. 3) циклов индентирования. В прямом цикле индентирования площадь контакта составляет $27.4 \mathrm{~mm}^{2}$ (часть 2), при отрыве площадь на $40 \%$ больше $-38.5 \mathrm{~mm}^{2}$ (часть 4). Такая существенная разница и обусловливает различие 

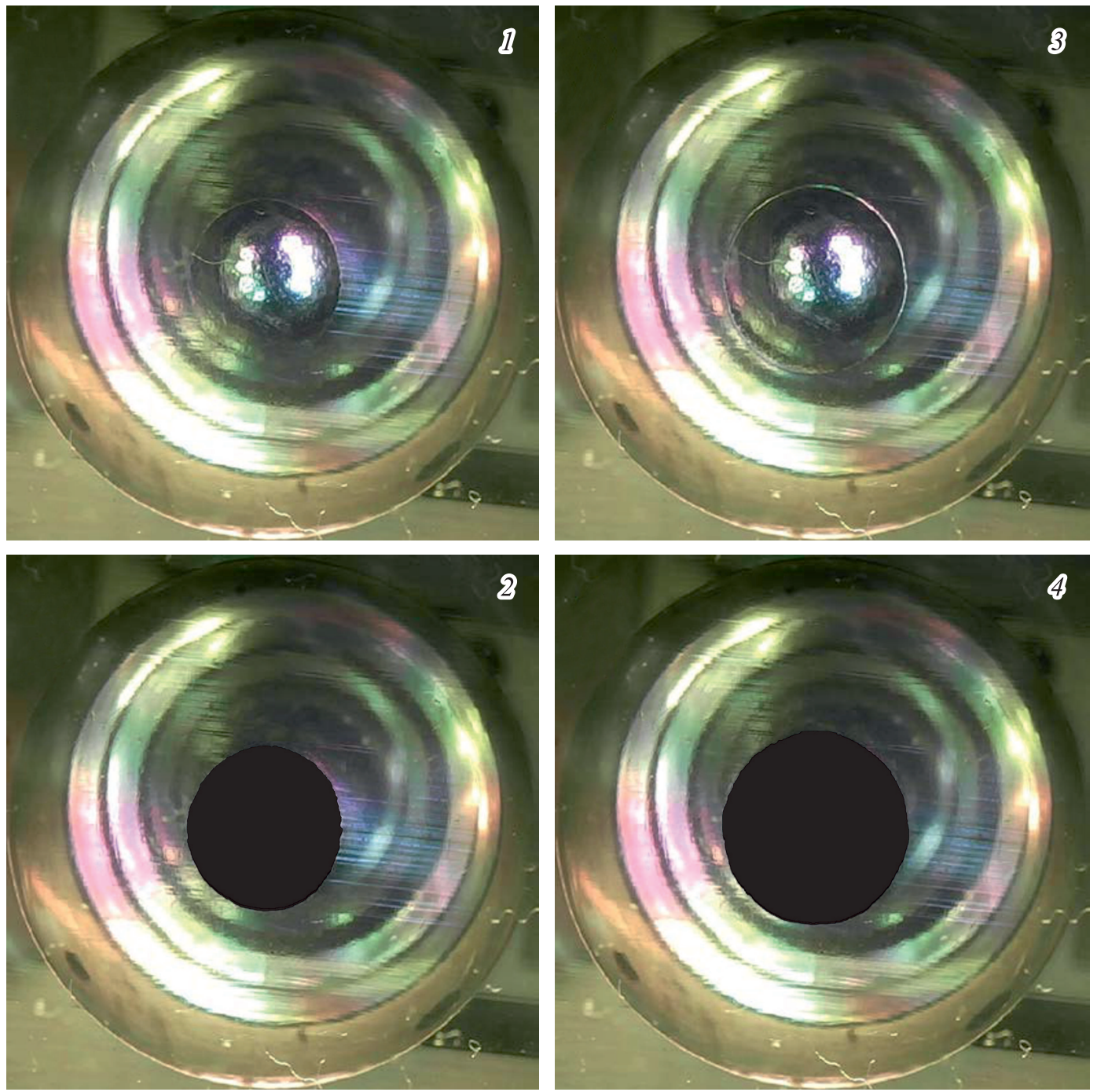

Рис. 3. Фотографии контактной области для глубины индентирования $d=0.2 \mathrm{~mm}$ в прямом цикле индентирования (части 1 и 2 ) и в процессе отрыва (части 3 и 4). Части 1 и 3 - это непосредственно снятые в эксперименте фотографии контактной области. Части 2 и 4 - те же самые фотографии, на которых в центре черным цветом выделена область контакта. Площадь контакта, показанная на части 2, составляет $27.4 \mathrm{~mm}^{2}$, на части $4-38.5 \mathrm{~mm}^{2}$. Фотографии соответствуют эксперименту, результаты которого показаны на рис. 2.

между нормальными силами при индентировании и отрыве за счет адгезионного вклада. В рассматриваемом случае $d=0.2 \mathrm{~mm}$ силы составляют $F_{N}=0.313 \mathrm{~N}$ в фазе индентирования и $F_{N}=0.291 \mathrm{~N}$ в фазе отрыва. Однако, как это показывает рис. 2, с уменьшением глубины индентирования $d$ обсуждаемое различие между силами существенно увеличивается. Поскольку адгезионная составляющая силы всегда направлена против упругой силы реакции резинового образца, при увеличении адгезионной составляющей значение измеряемой прибором силы уменьшается. Неодинаковое распространение площади контакта при прямом и обратном циклах индентирования (гистерезис площади) приводит к гистерезису силы, показанному на рис. 2. Отметим, что в цикле отрыва (часть 3 на рис. 3) граница контакта более выражена. Логично предположить, что при движении назад на границе контакта материал резины „подтянут“ к поверхности индентора, за счет чего образуется своеобразный мениск - „адгезионная шейка“. Преломление света за счет этого мениска дает четкое изображение границы. В начальной фазе индентирования такого мениска практически нет, поэтому область контакта гораздо хуже визуализирована (часть 1 на рис. 3). В работе [12] мы описали подобный эксперимент, в котором осуществлялось подряд три цикла индентирования, при этом все три зависимости показали аналогичное (описанному выше) поведение. Поэтому для рассматриваемой системы такое поведение - типичный случай.

Для сравнения полученных экспериментальных результатов с теоретическими моделями необходимо знать упругие параметры используемой резины. Резина является эластомером и почти не сжимаема, поэтому коэф- 
фициент Пуассона мы приняли равным $v=0.47$, близким к значению 0.5. В работе [12] в результаты серии экспериментов по индентированию цилиндрических инденторов с плоским основанием в аналогичный материал мы экспериментально определили, что используемая резина имеет модуль упругости $E \approx 0.324 \mathrm{MPa}$. Работа адгезии в паре резина-сталь на единицу площади зависит от многих факторов, но в проведенном эксперименте она может быть оценена значением $\gamma_{12} \approx 0.326 \mathrm{~J} / \mathrm{m}^{2}$. При теоретических оценках будем использовать эти величины.

В проведенном эксперименте (рис. 2) толщина эластомера составляла $h=25 \mathrm{~mm}$. При этом в области, в которой происходит разрушение контакта, контактный радиус а существенно меньше, чем толщина резинового образца $h$. Для рассматриваемого случая контакта жесткого индентора с упругим эластомером конечной толщины, находящимся на жесткой подкладке, в пределе, в котором толщина эластомера многократно превышает радиус контакта $\varepsilon=a / h \ll 1$, существует приближенное аналитическое решение адгезионной задачи [13]:

$$
\begin{aligned}
F= & \frac{4 E^{*} a^{3}}{3 R}\left(1-\varepsilon^{3} \frac{8 a_{1}}{3 \pi}\right)\left\{1-\frac{3 R}{2 E^{*} a^{2}} \sqrt{2 \pi E^{*} a \gamma_{12}}\right\}, \\
d & =\frac{a^{2}}{R}\left\{1-\varepsilon \frac{4 a_{0}}{3 \pi}-\varepsilon^{3} \frac{16 a_{1}}{5 \pi}\right. \\
& +\varepsilon^{4} \frac{32 a_{0} a_{1}}{9 \pi^{2}}-\frac{R}{E^{*} a^{2}} \sqrt{2 \pi E^{*} a \gamma_{12}} \\
& \left.\times\left(1-\varepsilon \frac{2 a_{0}}{\pi}-\varepsilon^{3} \frac{16 a_{1}}{3 \pi}+\varepsilon^{4} \frac{16 a_{0} a_{1}}{3 \pi^{2}}\right)\right\},
\end{aligned}
$$

где коэффициенты задаются равенствами

$$
a_{m}=\frac{(-1)^{m}}{2^{2 m}(m !)^{2}} \int_{0}^{\infty} L(u) u^{2 m} \mathrm{~d} u,
$$

$$
\begin{aligned}
& L(u)= \\
& =\frac{2(4 v-3) e^{-4 u}-\left[1+(4 v-3)^{2}+4 u+4 u^{2}\right] e^{-2 u}}{(4 v-3)-\left[1+(4 v-3)^{2}+4 u^{2}\right] e^{-2 u}+(4 v-3) e^{-4 u}},
\end{aligned}
$$

а редуцированное значение модуля сдвига определяется как $E^{*}=E /\left(1-v^{2}\right)$. Можно использовать и более простые выражения теории JKR для полупространства. Однако, поскольку эксперимент проводится с листом резины ограниченной толщины, решение (1)-(4) даст более точный результат.

Как уже указывалось выше, в фазе индентирования (экспериментальная кривая 1 на рис. 2) адгезия играет несущественную роль, поэтому для этой фазы логично провести расчет по формулам (1)-(4) для безадгезионного контакта, т. е. использовать при расчетах значение $\gamma_{12}=0 \mathrm{~J} / \mathrm{m}^{2}$. Результат такого расчета показан на рис. 2 штриховой кривой 3. Фактически это решение представляет безадгезионный упругий контакт Герца [14], обобщенный на случай эластомера конечной толщины. Как видно из сравнения кривых, безадгезионная кривая 3 лежит немного выше, чем экспериментальная кривая 1. Это означает, что в фазе индентирования наблюдается влияние адгезии, которая уменьшает упругую силу. Поскольку влияние адгезии довольно мало, теоретически рассчитанная безадгезионная кривая и экспериментально найденная зависимости проходят рядом. Штриховая кривая 4 - это зависимость, рассчитанная по формулам (1)-(4) при определенном в работе [12] значении работы адгезии $\gamma_{12} \approx 0.326 \mathrm{~J} / \mathrm{m}^{2}$. Видно, что эта кривая находится в области экспериментальных результатов, показанных линией 2.

Наибольшие отклонения расчетных результатов от экспериментальных для кривых 2 и 4 на рис. 2 наблюдаются в области больших глубин индентирования $d$ (рис. 2,a) и в области разрушения адгезионного контакта при $d<0$. Поскольку аппроксимация (1)-(4) работает хорошо только в пределе $a / h \ll 1$, то с увеличением радиуса контакта точность используемого аналитического решения уменьшается. С этим фактом может быть связано отклонение между теоретическими и экспериментальными результатами при больших значениях глубины индентирования $d$. Проведем дополнительно моделирование процесса индентирования методом граничных элементов, который позволяет для контакта индентора и эластомера конечной толщины численно найти точное решение задачи при адгезионном взаимодействии типа JKR [15]. Результаты такого моделирования показаны на рис. 2 символами-кружками, причем они во всем диапазоне глубин индентирования показывают хорошее совпадение с расчетом по формулам (1)-(4). Поэтому в рассматриваемой ситуации нарушением предела $a / h \ll 1$ с увеличением глубины индентирования $d$ нельзя объяснить отклонение экспериментальных результатов от расчетных. Однако сам подход JKR, несмотря на то, что его очень часто используют для интерпретации экспериментальных результатов, является лишь частным случаем общей теории адгезионного взаимодействия $[16,17]$. К тому же в эксперименте всегда существуют неучтенные эффекты. Учитывая эти моменты, полученное нами соответствие теории и эксперимента является достаточно убедительным, поскольку мы не использовали никакие подгоночные параметры. Такое совпадение результатов можно считать подтверждением предложенного в работе [13] аппроксимационного решения, а также методики численного моделирования [15].

Отдельного обсуждения требует отклонение экспериментальных и теоретических результатов в области отрицательных глубин индентирования $d<0$ (рис. 2,b). Здесь между теорией и экспериментом наблюдается качественное расхождение результатов. Отметим, что эксперимент был проведен при движении индентора с постоянной скоростью, т. е. в условиях контролируемого перемещения. 
В теории и численном моделировании контакт исчезает после возникновения контактной неустойчивости, как только величина удаления индентора от полупространства превышает критическое значение. Этот переход разрушения контакта в условиях контролируемого перемещения на рис. 2, $b$ показан стрелкой вверх. Однако кривая 2 на рис. $2, b$ демонстрирует существенное отличие экспериментального поведения от предсказываемого теоретически в области разрушения адгезионного контакта. Такая особенность наблюдалась во многих экспериментальных работах $[8,10,18]$. Многие исследователи объясняют такой механизм разрушения контакта наличием у поверхностей шероховатостей. Мы можем частично подтвердить этот вывод, поскольку провели серию экспериментов с шероховатыми поверхностями и выяснили, что величина гистерезиса сил в фазах индентирования и отрыва, а также адгезионная прочность контакта существенно зависят от характеристик шероховатости поверхности индентора [19]. Зависимость указанных величин от шероховатости подтверждает компьютерное моделирование [20-22], эксперимент [23] и теория [24]. Наличием шероховатостей объясняют также принципиальное различие зависимостей $F_{N}(d)$ в фазах индентирования и отрыва [20,23]. Однако в нашем эксперименте использовался отшлифованный до зеркального состояния индентор. На фотографиях контакта на рис. 3 на нем видны отражения светодиодов освещения и элементов объектива камеры. Несомненно, все поверхности имеют шероховатости $[25,26]$, а механическая шлифовка и полировка лишь уменьшает их размер. Однако сложно говорить о том, что шероховатости в случае зеркально отполированного индентора приводят к настолько существенному различию между зависимостями $F_{N}(d)$ в фазах индентирования и отрыва, как это показано кривыми 1 и 2 на рис. 2. Существуют феноменологические модели, объясняющие гистерезисное поведение за счет введения эффективной поверхностной энергии, отличающейся от теории JKR, например, [10]. Кривые 1 и 2 на pис. 2 можно получить в приближении теории JKR, если считать, что эффективная поверхностная энергия в фазе индентирования намного меньше, чем в фазе отрыва. Но такой подход не дает ответа на вопрос о причинах такого поведения. Одним из объяснений различия контактных свойств в фазах индентирования и отрыва может быть наличие некой „статической силы трения“ при распространении контакта. Такая „сила трения“ всегда создает сопротивление, которое в фазе индентирования не дает контакту распространиться до равновесного значения, задаваемого теорией. В фазе отрыва эта „сила трения“ действует обратным образом, т.е. существенно замедляет темп разрушения контакта. В итоге наблюдается описанный выше гистерезис [21]. Другим объяснением может быть влияние поверхностного натяжения на поверхности резины $[27,28]$, которое способно как усилить, так и ослабить адгезию. Но в любом случае причины такого резкого различия контактных свойств в фазах индентирования и отрыва на сегодняшний день до конца не выяснены и для дальнейшего понимания необходимо продолжать соответствующие исследования.

\section{3. Влияние продолжительности контакта на адгезионную прочность}

При проведении экспериментов по изучению адгезионных свойств для выяснения влияния какого-либо одного фактора, такого как скорости сдвига, шероховатости поверхности, геометрической формы индентора и т. П., необходимо провести большое количество экспериментов, в которых должны быть фиксированными все другие параметры. При этом довольно сложно сохранять одинаковым значение поверхностной энергии (удельной работы сил адгезии) контактирующих поверхностей, поскольку после контакта индентора с резиной на его поверхности остаются частицы резины и поверхностная энергия существенно уменьшается. Поэтому после каждого эксперимента необходимо одинаковым образом очищать контактирующие поверхности, но даже это не гарантирует постоянного значения поверхностной энергии. Вторым фактором, влияющим на поверхностную энергию, является продолжительность контакта. В этом разделе статьи мы описываем результаты проведенного нами экспериментального исследования продолжительности контакта на его адгезионную прочность, в котором в качестве эластомера использовалась существенно более мягкая резина TARNAC CRG N0505, чем TARNAC CRG N3005, результаты для которой приведены на рис. 2.

В ходе эксперимента в лист резины CRG N0505 толщиной $h=5 \mathrm{~mm}$ индентировался отполированный до зеркального состояния сферический индентор, имеющий радиус $R=40 \mathrm{~mm}$, на глубину $d_{\max }=0.1 \mathrm{~mm}$. После достижения указанной глубины индентор оставался без движения в течение времени $t$, после чего сдвигался в обратном направлении до полного исчезновения контакта. В фазах индентирования и отрыва скорость движения индентора была одинаковой и составляла $v=0.2 \mu \mathrm{m} / \mathrm{s}$. Перед каждым последующим экспериментом поверхность индентора очищалась, поскольку резина CRG N0505 после продолжительного контакта оставляет на инденторе пятно маслянистого вида, что существенно снижает поверхностную энергию при последующих индентированиях (более жесткая резина CRG N3005 гораздо меньше загрязняла поверхность). Результаты описанного эксперимента приведены на рис. 4. На рис. 4, а показаны зависимости нормальной силы $F_{N}$ от глубины индентирования $d$. Во всех проведенных экспериментах индентирование осуществлялось на одну и ту же глубину $d_{\max }=0.1 \mathrm{~mm}$. На рис. 4, $a$ видно, что фазы индентирования и отрыва существенно отличаются, как это уже было ранее показано на рис. 2 для другого эксперимента. На рис. 4, $a$ для всех кривых в положении покоя в течение времени 

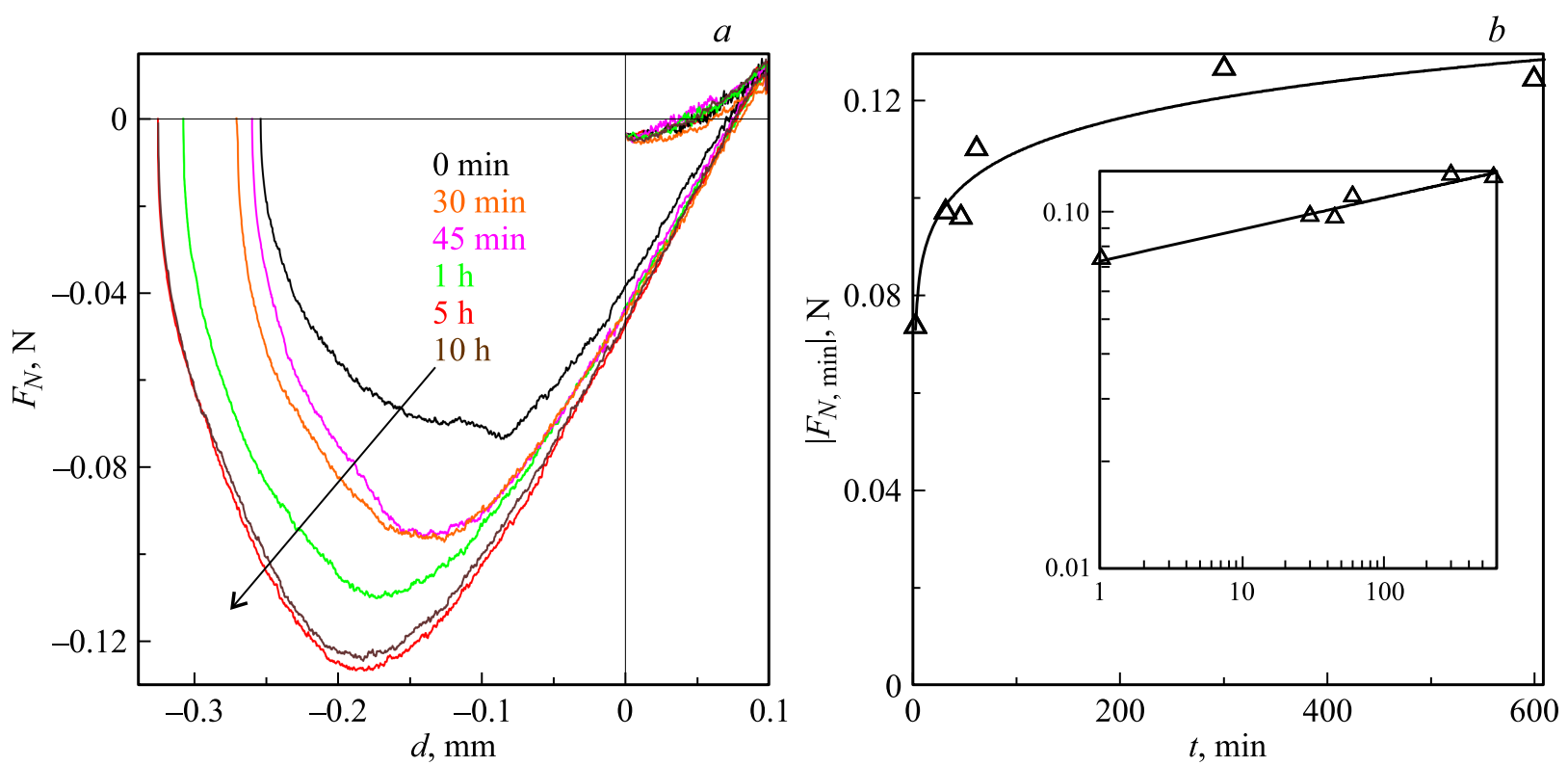

Рис. 4. $a-$ зависимости нормальной силы $F_{N}$ от глубины индентирования $d$ для индентирования сферического индентора, имеющего радиус $R=40 \mathrm{~mm}$, в лист мягкой резины TARNAC CRG N0505 толщиной $h=5 \mathrm{~mm}$. Приведенные кривые соответствуют различным временам покоя индентора при максимальной глубине индентирования, которые указаны на рисунке. В среднем со временем ожидания увеличивается прочность контакта на отрыв, что показано на рисунке стрелкой; $b-$ зависимость максимального абсолютного значения нормальной силы $F_{N}$ в фазе отрыва от времени ожидания $t$, на вставке к рисунку показана аналогичная зависимость в двойных логарифмических координатах.

ожидания (максимальное время составляло $10 \mathrm{~h}$ ) нормальная сила изменяется слабо. Это говорит о том, что область контакта в состоянии покоя расширяется не существенно, что подтверждает выдвинутую выше гипотезу о существовании „силы трения“, результатом действия которой является гистерезис нормальной силы при прямом и обратном циклах индентирования. На рис. $4, b$ показана зависимость максимального абсолютного значения нормальной силы на стадии отрыва $\left|F_{N \text {, min }}\right|$ от времени покоя $t$. Сила $\left|F_{N \text {, min }}\right|$ представляет адгезионную прочность контакта — чтобы разрушить контакт, необходимо приложить как минимум эту силу. На рисунке символы-треугольники соответствуют экспериментальным результатам, а сплошная кривая показывает аппроксимацию степенной функцией

$$
\left|F_{N, \min }\right|=0.07293 t^{0.0883} \text {, }
$$

где время $t$ выражено в минутах. Поскольку первая точка на графике на рис. 4, $b$ получена для времени ожидания $t=0$, а производится аппроксимация степенной функцией (5), вместо нуля здесь было взято близкое в условиях эксперимента к нулю значение $t=1 \mathrm{~min}$. Зная вид зависимости (5), можно оценить изменение поверхностной энергии исходя из сопоставления с классической теорией JKR, которая дает минимальное значение нормальной силы в области разрушения контакта в виде

$$
F_{N, \min }=-\frac{3}{2} \pi \gamma_{12} R
$$

где $R$ - радиус индентора. При сопоставлении формул (5) и (6) легко найти зависимость поверхностной энергии $\gamma_{12}$ от времени контакта $t$. Такую экспериментально определенную зависимость можно использовать при моделировании контактных явлений методом граничных элементов [15], а для аксиально симметричных тел возможно использование более простых методов типа метода редукции размерности [29,30]. Моделирование с учетом изменения поверхностной энергии позволит описать эволюцию адгезионной прочности контакта и его геометрической конфигурации во времени. Очевидно, что учет зависимости поверхностной энергии от времени позволит получить нетривиальные результаты для реверсивного адгезионного контакта.

Отметим, что непостоянство поверхностной энергии создает дополнительные сложности при интерпретировании экспериментальных результатов. С одной стороны, с ростом скорости движения индентора увеличивается влияние вязкоупругих эффектов, что не позволяет считать контакт квазистатическим. С другой стороны, при малой скорости индентор находится более продолжительное время в контакте, а поверхностная энергия неоднородно распределена по его поверхности, поскольку ее значение зависит от того, как долго та или иная часть индентора контактировала с эластомером. Поэтому сравнивать результаты экспериментов можно только в том случае, если при их проведении используется оптимальная промежуточная скорость, позволяющая свести к минимуму эти два эффекта. Отметим, 
что для мягкой резины TARNAC CRG N0505 (рис. 4, a) после точки первого контакта индентора с поверхностью резины контакт за счет адгезионного взаимодействия распространяется гораздо дальше по поверхности индентора, чем в случае использования жесткой резины TARNAC CRG N3005, которая использовалась в экспериментах, проиллюстрированных на рис. 2 и 3. Указанная тенденция сохранялась для всех проведенных экспериментов.

\section{4. Зависимость прочности адгезионного контакта от его предыстории}

В предыдущем подразделе настоящей работы было выяснено, каким образом адгезионная прочность зависит от времени контакта при других постоянных условиях. Изучим теперь зависимость адгезионной прочности от глубины индентирования, поскольку многие экспериментальные работы показывают наличие такой зависимости, например, [8]. Для выяснения влияния глубины индентирования на адгезионную прочность была проведена серия экспериментов по индентированию сферического индентора, имеющего радиус $R=33 \mathrm{~mm}$ (этот же индентор использовался для получения результатов, приведенных на рис. 2) в лист жесткой резины TARNAC CRG N3005 толщиной $h=5 \mathrm{~mm}$. Во всех тестах индентирование производилось с одинаковой скоростью $v=1 \mu \mathrm{m} / \mathrm{s}$ на максимальную глубину $d_{\max }$, после чего сразу совершалось движение в обратном направлении до полного исчезновения контакта. В проведенной серии экспериментов изменялась только глубина индентирования $d_{\text {max }}$. На рис. 5 показаны полученные результаты. Всего было выполнено 7 экспериментов, в которых глубина $d_{\max }$ изменялась от 0.1 до $0.7 \mathrm{~mm}$ с шагом $0.1 \mathrm{~mm}$. Для каждого значения $d_{\max }$ подряд проводилось три цикла индентирования, между которыми поверхность индентора не очищалась. Очистка поверхности производилась только между экспериментами с различными значениями $d_{\max }$.

На рис. 5, а показаны измеренные зависимости нормальной силы $F_{N}$ от глубины индентирования $d$. На этом рисунке для каждого значения $d_{\max }$ показаны три кривые, поскольку во всех случаях проводилось по три цикла индентирования. Из рисунка следует, что при увеличении величины $d_{\max }$ прочность адгезионного контакта возрастает. Во всех экспериментах использовался один и тот же индентор, отшлифованный до состояния зеркала в видимом диапазоне спектра, поэтому он имел минимальные шероховатости. Как видно из рисунка, в рассматриваемом случае зеркально гладкого индентора глубина, на которую производится индентирование, оказывает существенное влияние на гистерезисные характеристики и адгезионную прочность контакта.

На рис. 5, $b$ для всех проведенных экспериментов показаны зависимости максимального абсолютного зна- чения адгезионной силы в области разрушения контакта $d<0$ от максимальной глубины индентирования $d_{\max }$. Здесь ромбами показаны значения для первого цикла индентирования, треугольниками - для второго и кружками - для третьего. Полученные результаты показывают отсутствие какой-либо устойчивой тенденции в изменении адгезионной прочности при различных циклах индентирования. Поэтому для определения зависимости силы адгезии от глубины индентирования проводилась аппроксимация с использованием всех значений, показанных на рис. $5, b$. В результате такой аппроксимации была получена функция

$$
\left|F_{N, \min }\right|=0.06853\left(d_{\max }\right)^{0.2827} .
$$

Зависимость (7) показана сплошными линиями на рис. 5, $b$, а также на вставке к этому рисунку в двойных логарифмических координатах. В результате можно заключить, что зависимости адгезионной прочности от продолжительности контакта (рис. 4, $b$, функция (5)) и от максимальной глубины индентирования (рис. $5, b$, функция (7)) являются степенными функциями. При этом, однако, стоит учитывать, что в эксперименте, результаты которого показаны на рис. 5, индентор сдвигается с довольно малой скоростью $v=1 \mu \mathrm{m} / \mathrm{s}$. Это означает, что с увеличением значения $d_{\max }$ увеличиваться время контакта. Например, при глубине индентирования $d_{\max }=0.1 \mathrm{~mm}$ общее контактное время $t$ составляет около $235 \mathrm{~s}$, а при $d_{\max }=0.7 \mathrm{~mm}$ продолжительность контакта уже более $1400 \mathrm{~s}$. Поэтому зависимость (7) может иметь место из-за увеличения с глубиной индентирования времени контакта, т.е. на самом деле она может быть функцией времени. Однако если записать (7) как функцию времени продолжительности контакта, то это не изменит значение показателя степени 0.2827 , поскольку общее время контакта увеличивается пропорционально глубине индентирования $d_{\max }$. В эксперименте, в котором изучалось влияние продолжительности контакта на адгезионную прочность, наблюдался существенно более низкий показатель степени 0.0883 (см. формулу (5)). То есть при варьировании времени контакта и глубины индентирования показатели степеней в соответствующих степенных функциях отличаются более чем в 3 раза. Этот факт позволяет говорить о действии различных механизмов увеличения адгезионной прочности в рассмотренных ситуациях. Судя по всему, в первом случае (рис. 4) адгезионная прочность увеличивается за счет упрочнения адгезионных связей со временем. Такое упрочнение может происходить ввиду протекания в контактной зоне химических реакций, в результате которых увеличивается работа адгезии по разрушению контакта. Однако увеличение адгезионной прочности частично может осуществляться также и за счет релаксации эластомера и заполнения резиной микроскопических шероховатостей. При таком заполнении увеличивается реальная площадь контакта, а значит и адгезионная прочность. Во втором случае (рис. 5) логично 

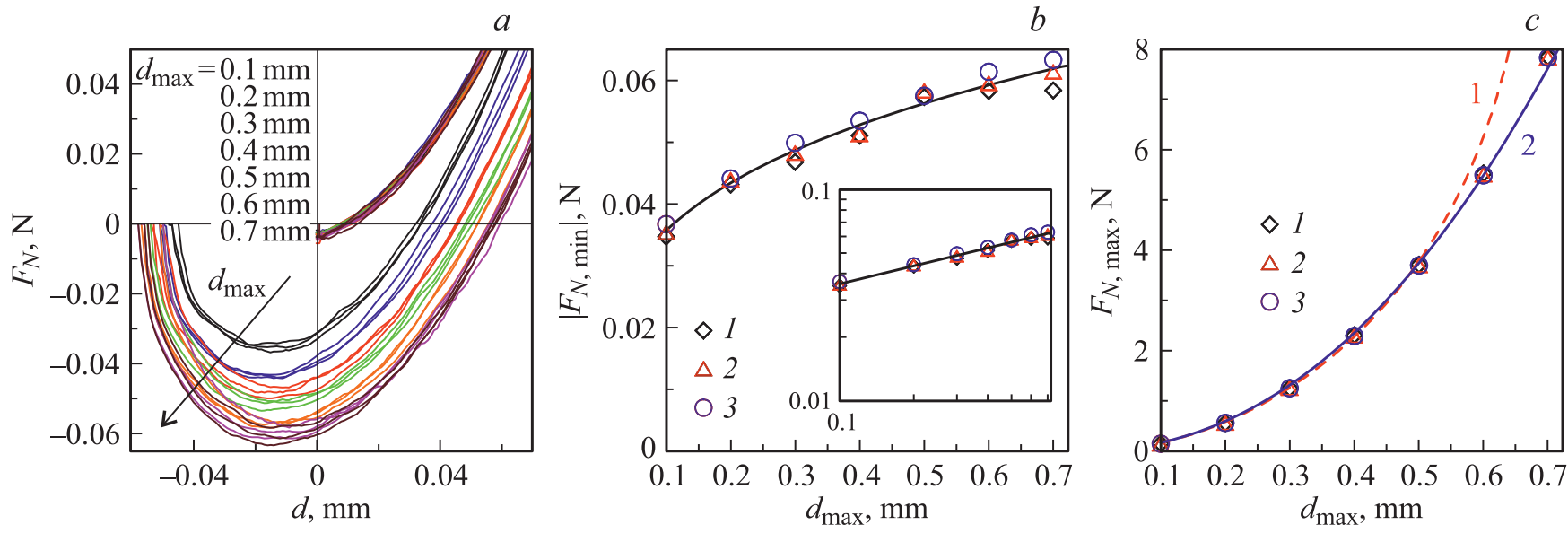

Рис. 5. $a-$ зависимости нормальной силы $F_{N}$ от глубины индентирования $d$ для индентирования сферического индентора, имеющего радиус $R=33 \mathrm{~mm}$, в лист твердой резины TARNAC CRG N3005 толщиной $h=5 \mathrm{~mm} ; b-$ зависимости максимального абсолютного значения силы адгезии в зоне разрушения контакта $d<0$ от максимальной глубины индентирования, на вставке показана аналогичная зависимость в двойном логарифмическом масштабе; $c$ - зависимости максимального значения нормальной силы от максимальной глубины индентирования во всем диапазоне измеряемых величин.

предположить, что адгезионная прочность в основном увеличивается из-за возрастания контактного давления при увеличении максимальной глубины индентирования: при большем давлении резина более плотно прилегает к поверхности индентора и при этом гораздо лучше заполняет микроскопические шероховатости. Таким образом, мы предполагаем, что в проиллюстрированном на рис. 4 случае доминирующий механизм увеличения адгезионной прочности заключается в протекании химических реакций в зоне контакта, а в случае, показанном на рис. 5, прочность контакта возрастает ввиду увеличения реальной площади с ростом давления.

На рис. 5, с символами дополнительно показаны зависимости максимальных нормальных сил $F_{N}$ для всех проведенных экспериментов, соответствующих максимальной глубине индентирования $d=d_{\max }$. Поскольку все эксперименты проводились в одинаковых условиях, несмотря на то, что на рис. 5, с показаны точки для различных экспериментов, все эти точки должны ложиться на единственную кривую $F_{N}(d)$, которая может быть рассчитана согласно процедуре (1)-(4). Найденная по указанным формулам кривая показана на рис. 5, штриховой линией 1. Эта зависимость была определена при параметрах эксперимента $v=0.47, E=0.324 \mathrm{MPa}$ и $h=5 \mathrm{~mm}$. Расчетная кривая соответствует безадгезионному случаю $\gamma_{12}=0 \mathrm{~J} / \mathrm{m}^{2}$, поскольку, как мы указывали ранее, в фазе индентирования адгезия практически не вносит вклад в нормальную силу. Сплошной кривой 2 на рис. 5, с показана зависимость $F_{N}(d)$, рассчитанная при помощи метода граничных элементов для двуслойных систем [15]. Из рисунка следует, что теоретическая модель (1)-(4) отклоняется от экспериментальных результатов, как только глубина индентирования (а значит и радиус контакта) превышает некоторое критическое значение. Данные, полученные в результате моделирова- ния методом граничных элементов (кривая 2), адекватно описывают экспериментальные результаты во всем диапазоне глубин индентирования. Вышеописанный рис. 2 также показывает хорошее совпадение эксперимента с результатами компьютерного моделирования во всем используемом диапазоне глубин индентирования для другой толщины резинового образца $h=25 \mathrm{~mm}$.

\section{5. Влияние на прочность адгезионного контакта степени очистки индентора}

В предыдущих разделах описаны два способа, с помощью которых можно изменять адгезионную прочность непосредственно во время контакта - она возрастает с увеличением времени контакта и глубины индентирования. Однако на значение поверхностной энергии и соответственно на адгезионную прочность также существенно влияет изначальный химический состав поверхности индентора. Во многих экспериментах большой проблемой при интерпретации полученных результатов является сложность контролирования поверхностной энергии. С целью определения влияния на адгезионную прочность способа обработки поверхности был проведен эксперимент. Сперва поверхность индентора обрабатывалась наждачной бумагой для создания ярко выраженной шероховатости. Поскольку целью не было выяснение влияния шероховатости, ее параметры мы не измеряли. Затем проводилась ультразвуковая очистка индентора в водяном растворе EMAG EM-404 (на 1.41 воды $40 \mathrm{ml}$ средства ЕM-404) с помощью ультразвукового прибора CD-4800 в течение $16 \mathrm{~min}$, после чего индентор высушивался струей сжатого воздуха. После указанных манипуляций поводился эксперимент индентирования в резиновый образец. 

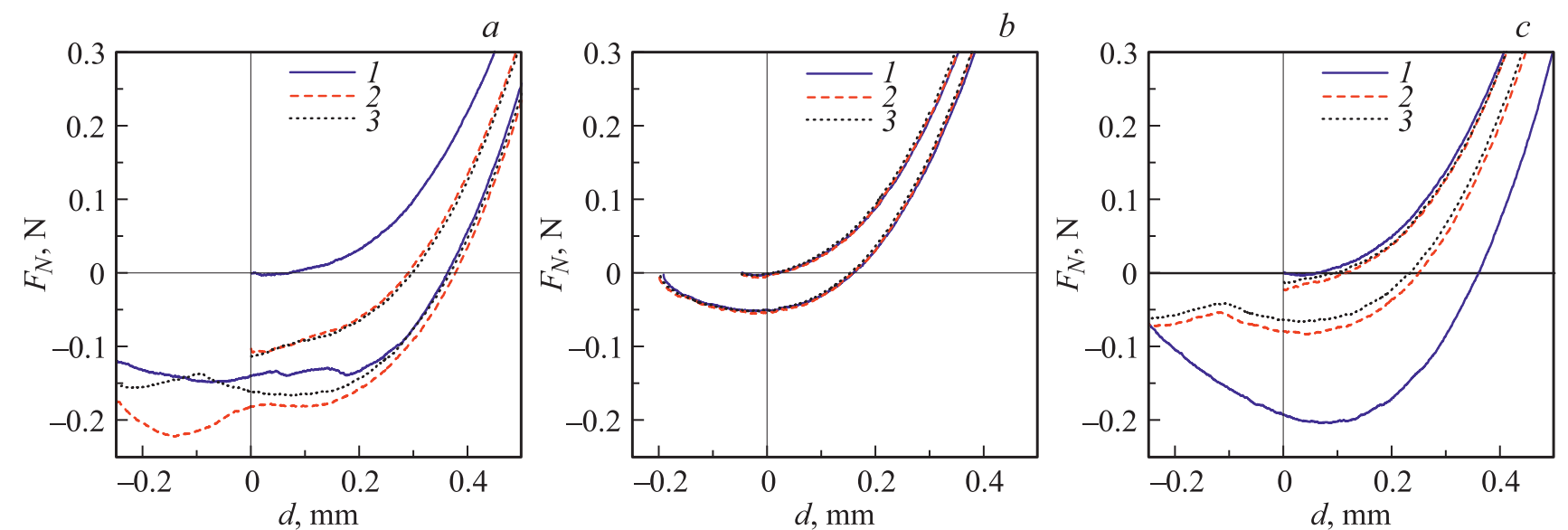

Рис. 6. Зависимости нормальной силы $F_{N}$ от глубины индентирования $d$ для индентирования шероховатого сферического индентора в лист резины TARNAC CRG N0505 толщиной $h=5 \mathrm{~mm}$.

Результаты проведенных экспериментов показаны на рис. 6. Здесь во всех рассмотренных случаях выполнялось подряд три цикла индентирования. Зависимости $F_{N}(d)$ для первого цикла показаны сплошными линиями, для второго - штриховыми, для третьего пунктирными. В эксперименте, результаты которого показаны на рис. 6, $a$, после обработки индентора наждачной бумагой он оставался на воздухе несколько суток, после чего выполнялась его ультразвуковая очистка и осушка, затем проводился эксперимент. В первом цикле индентирования кривая $F_{N}(d)$ для фазы вдавливания начинается вблизи начала координат, поскольку в фазе индентирования адгезионное взаимодействие оказывает незначительное влияние. Интересной особенностью проведенного эксперимента является то, что в фазе отрыва все зависимости $F_{N}(d)$ на рис. 6, $a$ имеют немонотонный вид. Это может быть связано как с ярко выраженной шероховатостью поверхности индентора, так и с химической неоднородностью поверхности, приводящей к неоднородному распределению поверхностной энергии. Отметим, что контакты между эластомером и шероховатыми поверхностями часто показывают немонотонный вид зависимости нормальной силы от глубины индентирования, что подтверждает компьютерное моделирование [20,21]. Это происходит потому, что при наличии шероховатостей (либо за счет химической неоднородности) на стадии разрушения контакта возникают устойчивые конфигурации, когда в некотором диапазоне глубин индентирования конфигурация контакта не изменяется или изменяется слабо. Подобная ситуация реализуется в случае инденторов с гладкой волнистой поверхностью [31,32]. Во всех экспериментах, результаты которых показаны на рис. 6, индентор приводился в контакт, затем погружался в резину на максимальную глубину $d=1.0 \mathrm{~mm}$, после чего сдвигался в обратном направлении до величины глубины индентирования $d=-0.25 \mathrm{~mm}$. Скорость движения индентора в обеих фазах составляла $v=0.2 \mu \mathrm{m} / \mathrm{s}$.
В ходе описанных манипуляций записывались значения нормальной силы и глубины индентирования, а также сохранялись фотографии области контакта. При достижении величиной $d=-0.25 \mathrm{~mm}$ индентор смещался к точке первоначального контакта $d=0.0 \mathrm{~mm}$, после чего сразу выполнялся следующий цикл индентирования. Однако в эксперименте, показанном на рис. 6, $a$, при достижении величины $d=-0.25 \mathrm{~mm}$ контакт не разрушался полностью, о чем свидетельствует отличное от нуля значение нормальной силы $F_{N}$ в этой точке для всех трех циклов индентирования в фазе отрыва. Поэтому второй и третий циклы индентирования хоть и начинались при нулевой глубине $d=0 \mathrm{~mm}$, при этом значении $d$ контактная область была гораздо больше, чем в первом цикле индентирования, за счет не полностью разрушенного контакта в предыдущем цикле. Это обусловливает отрицательное значение $F_{N}<0$ в начале фазы индентирования при $d=0 \mathrm{~mm}$ во втором и третьем циклах. На рис. 6, $a$, видно, что все три кривые $F_{N}(d)$ для фазы отрыва являются немонотонными, что говорит о наличии в фазе отрыва индентора устойчивых контактных конфигураций. Причем эти устойчивые конфигурации при каждом последующем цикле индентирования возникают при различных $d$, поскольку все три кривые отличаются друг от друга. Такое различие осуществляется за счет изменения вида распределения поверхностной энергии по зоне контакта при взаимодействии индентора с материалом резины.

После эксперимента, результаты которого показаны на рис. $6, a$, индентор более суток оставался на воздухе, после чего без его очистки было снова проведено подряд три цикла индентирования, которые показаны на рис. $6, b$. Здесь кривые $F_{N}(d)$ для всех циклов практически совпадают. Из этого можно сделать вывод о том, что распределение поверхностной энергии по поверхности индентора в ходе этого эксперимента не изменяется. Для случая, показанного на рис. $6, b$, значение адгезионной прочности гораздо меньше, чем это показывает рис. $6, a$. 
Это связано с наличием остатков резины на поверхности индентора после первого эксперимента и с окислением его поверхности, поскольку оба фактора приводят к уменьшению поверхностной энергии.

Последняя серия экспериментов (рис. 6,c) была проведена после очередной очистки поверхности индентора в ультразвуковой ванне. Этот эксперимент во многом повторяет поведение, показанное на рис. $6, a$, поскольку здесь так же не происходит полного разрушения контакта. Однако здесь в первом цикле индентирования в фазе отрыва отсутствуют устойчивые контактные конфигурации, чего нельзя сказать о двух последующих циклах, для которых кривые $F_{N}(d)$ являются немонотонными.

Все три эксперимента, показанные на рис. 6, были проведены с одним и тем же индентором, т.е. его шероховатость не изменялась от эксперимента к эксперименту. При этом в некоторых случаях кривые $F_{N}(d)$ показывают существование устойчивых конфигураций контакта в процессе отрыва, в иных ситуациях таких устойчивых конфигураций не возникает. На такое качественное различие в поведении влияет то, что на поверхности индентора после каждого цикла индентирования остаются частицы резины, которые из-за влияния шероховатостей неоднородно распределены. Наличие следов резины на инденторе приводит к уменьшению поверхностной энергии, причем неоднородно по области контакта. Кроме того, поверхность индентора вступает в химические реакции с резиной и воздухом (окисление), что также приводит к изменению поверхностной энергии.

Таким образом, вариации поверхностной энергии существенно влияют на контактное поведение шероховатых поверхностей качественным образом. Мы предполагаем, что от характера распределения поверхностной энергии зависит наличие либо отсутствие устойчивых контактных конфигураций в фазе отрыва индентора. Отметим, что в классической теории JKR параметр поверхностной энергии влияет на поведение адгезионного контакта лишь количественно [11]. В случае же контакта шероховатых поверхностей от поверхностной энергии качественно зависит вид кривой $F_{N}(d)$, а значит и свойства адгезионного контакта $[23,24]$. Стоит отметить, что в настоящее время адгезионный контакт между шероховатыми поверхностями является предметом оживленной дискуссии в ведущих мировых научных группах. Несмотря на большое количество экспериментальных и теоретических работ последних лет в этом направлении [8,20-23,33-36], здесь все еще остается много нерешенных вопросов. Отметим, что при проведении серии экспериментов по исследованию адгезии шероховатых поверхностей важно стремиться сохранять постоянными условия эксперимента - использовать одну и ту же сталь для изготовления инденторов, одинаковые условия ультразвуковой очистки и обработки поверхности инденторов, одинаковые температурные и скоростные режимы и т.д. Все перечисленные факторы существенно влияют на адгезионные свойства контакта.
Как показано в этом подразделе, адгезионная прочность контакта за счет окисления поверхности индентора сильно зависит от времени нахождения индентора на воздухе. При этом мы не исследовали влияния времени, в течение которого индентор находится на воздухе, на адгезионную прочность. Такое исследование требует выполнения серии дополнительных экспериментов, поэтому мы планируем провести его в будущих работах.

\section{6. Обсуждение особенностей экспериментов в условиях контролируемой силы}

Во всех описанных выше экспериментах индентор сдвигался с постоянной скоростью (условия контролируемого перемещения). В технических приложениях для разрушения контакта часто прикладывают постепенно увеличивающуюся силу. Известно, что в условиях контролируемой силы и контролируемого перемещения разрушение адгезионного контакта происходит на различных участках зависимости $F_{N}(d)$ [11]. На рис. 7 показаны результаты проведенного эксперимента в условиях контролируемой силы. На рис. 7, a приведены зависимости для случаев жесткого соединения индентора с приводом, на рис. $7, b$ - полученные при соединении индентора с приводом жесткой пружиной, на рис. 7,c - мягкой пружиной. На рисунке показаны зависимости не от глубины индентирования $d$, а от величины сжатия $(x>0)$ и натяжения $(x<0)$ пружины. На рис. 7, $a$ реализовано жесткое соединение индентора с приводом, поэтому в этом случае $x \equiv d$. Индентор в этом эксперименте сдвигался с постоянной скоростью $v=0.5 \mu \mathrm{m} / \mathrm{s}$. Здесь реализуется ситуация, во многом аналогичная показанной на рис. 2, поскольку используется тот же самый индентор и тот же резиновый образец. Однако в эксперименте, результаты которого показаны на рис. 7, толщина резинового листа составляла $h=5 \mathrm{~mm}$ (на рис. 2 показан случай с толщиной $h=25 \mathrm{~mm}$ ). Потому на тех же глубинах индентирования нормальная сила на рис. 7 гораздо больше, чем на рис. 2. В ситуации, показанной на рис. $7, b$, индентор был присоединен к приводу с помощью пружины, поэтому здесь $x$ - смещение верхнего конца пружины. Поскольку целью исследования является определение влияние жесткости соединения индентора и пружины, должны быть созданы условия, в которых нормальная сила нарастает приблизительно с одинаковой скоростью, вне зависимости от жесткости пружины. Общее время фазы индентирования на рис. 7, $a$ составляет $t=x / v=400 \mathrm{~s}$. Для двух других случаев необходимо, чтобы это время также было порядка $400 \mathrm{~s}$. При этом индентирование следует производить до такого же значения нормальной силы, как и на рис. 7, $a$, т. е. приблизительно до величины $F_{N}=0.6 \mathrm{~N}$. Для реализации таких условий в эксперименте, результаты которого показаны на рис. 7, $b$, выбиралась скорость 

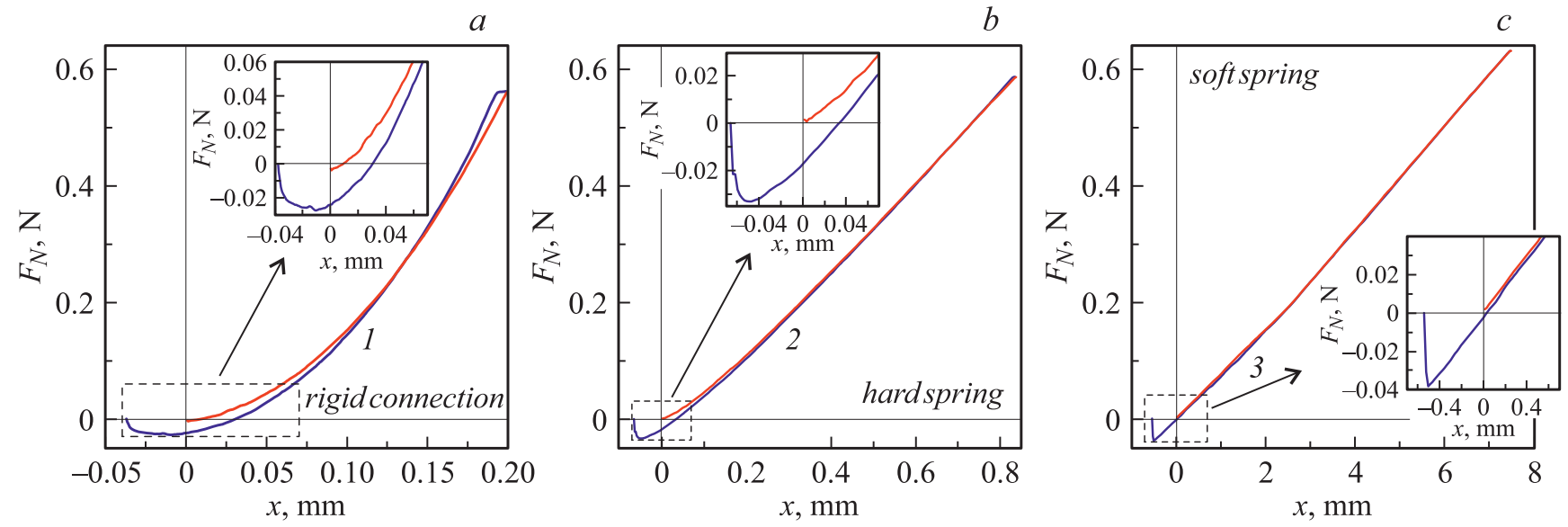

Рис. 7. Зависимости нормальной силы $F_{N}$ от перемещения верхнего конца пружины $x$, к которой прикреплен индентор, для индентирования сферического индентора, имеющего радиус $R=33 \mathrm{~mm}$, в лист резины TARNAC CRG N3005 толщиной $h=5 \mathrm{~mm}$. На вставках к рисунку показаны увеличенные фрагменты зависимостей, обозначенные на рисунках прямоугольниками из штриховых линий.
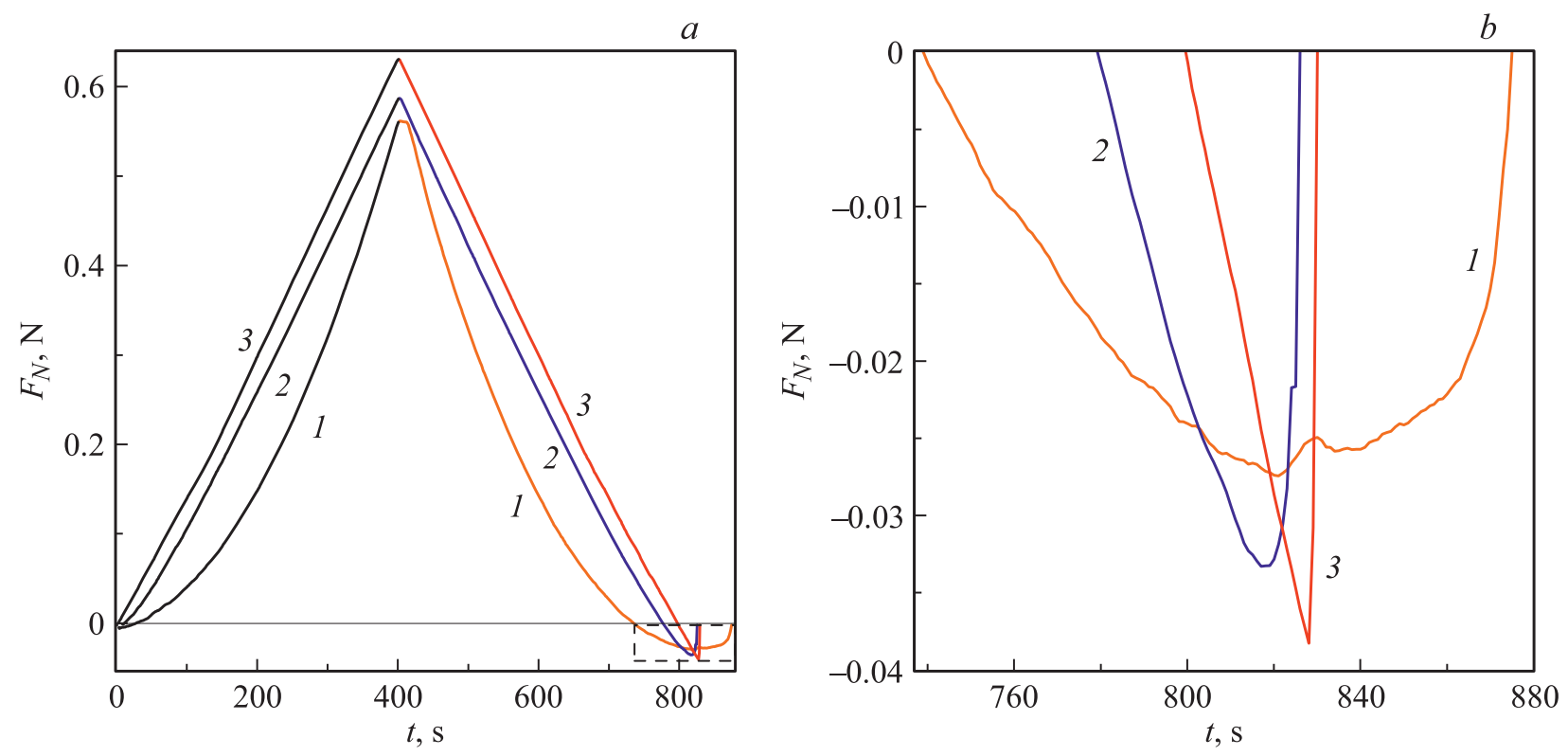

Рис. 8. $a-$ зависимости нормальной силы $F_{N}$ от времени $t$, соответствующие эксперименту, результаты которого показаны на рис. $7 ; b-$ увеличенная область рис. $8, a$, показанная на нем в нижнем правом углу штриховыми линиями.

движения верхнего конца пружины $v=2.125 \mu \mathrm{m} / \mathrm{s}$, а для рис. 7, с скорость составляла уже $v=18.75 \mu \mathrm{m} / \mathrm{s}$. Перед экспериментами, показанными на рис. $7, b$ и $c$, сначала определялась глубина индентирования, на которой реализуется такая же нормальная сила, как и на рис. 7, $a$, а затем уже рассчитывалась необходимая скорость, чтобы достичь этой силы за отведенное на процесс индентирования время $t=400 \mathrm{~s}$. Отметим, что в случае жесткой (рис. 7,b) и мягкой (рис. 7,c) пружин в области вдавливания зависимость $F_{N}(x)$ близка к прямой линии, что позволяет рассчитать жесткость системы контакт-пружина как угол наклона этой линии. Так, для жесткой пружины мы получили $k=768 \mathrm{~N} / \mathrm{m}$, а для мягкой пружины жесткость $k=88.8 \mathrm{~N} / \mathrm{m}$, что почти в 10 раз меньше. Ситуацию, показанную на рис. 7, $a$, также можно считать контактом с пружиной, только с гораздо большей жесткостью. На всех трех рисунках максимальная нормальная сила приблизительно одинакова, поэтому максимальная глубина индентирования индентора $d$ также приблизительно одинакова, только рис. 7, $a$ показывает непосредственно глубину индентирования, а на других рисунках ее необходимо определять косвенным образом. Это можно легко сделать, поставив в соответствие каждому значению силы $F_{N}$ на рис. 7, $b$ и $c$ величину глубины индентирования $d$, определяя ее из зависимости $F_{N}(x)$, показанной на рис. 7, $a$, поскольку для показанного на этом рисунке случая $x \equiv d$. 
Важным результатом проведенного эксперимента является то, что на описанных выше рисунках наблюдается четкая тенденция - в области разрушения контакта с уменьшением жесткости пружины контакт разрушается более быстро и резко. В случае, показанном на рис. 7, $a$, реализуются условия контролируемого смещения индентора, поэтому контакт исчезает монотонно, переходя через устойчивые состояния. Подобное поведение прослеживалось во всех описанных выше экспериментах, поскольку до этого использовались условия контролируемого перемещения. Рис. 7, $c$ показывает, что при превышении глубиной индентирования критического значения контакт исчезает практически мгновенно. Это связано с тем, что в условиях контролируемой силы на контакт всегда действует сила от натянутой пружины. При этом если контакт становится неустойчивым, пружина быстро вытягивает индентор из контакта. Для обеспечения условий контролируемой силы пружина должна быть довольно мягкой, чтобы изменение ее натяжения при вытягивании индентора не привело к уменьшению действующей со стороны пружины нормальной силы. Это возможно только в случае, если перемещение индентора при разрушении контакта существенно меньше натяжения пружины, а чем мягче пружина, тем большую величину натяжения она имеет при фиксированной нормальной силе.

На рис. 8 показаны те же зависимости нормальной силы $F_{N}$, что и на рис. 7 , только не от координаты пружины $x$, а от времени процесса индентирования $t$. Причем рис. $8, b$ представляет увеличенную часть рис. $8, a$ в зоне отрицательных значений нормальной силы $F_{N}$, где контакт существует только за счет адгезии. Здесь кривые, пронумерованные от 1 до 3 , соответствуют номерам кривых на рис. 7. Из рис. 8, $a$ следует, что вначале на протяжении времени $t=400 \mathrm{~s}$ длится стадия вдавливания индентора, после чего начинается его движение в обратную сторону. Указанное движение осуществляется либо за счет непосредственного перемещения индентора (кривая 1), либо за счет ослабления сжатия прижимающей пружины (кривые 2 и 3). На рис. 8, $b$ видно, что в случае мягкой пружины (кривая 3) контакт исчезает скачкообразно, как это предсказывает теория адгезионного взаимодействия JKR [11]. К недостаткам экспериментов, проводимых в условиях контролируемой силы, можно отнести проявление эффектов вязкоупругости. Случай контролируемой силы представляет динамическую задачу, при этом в точке исчезновения контакта при контролируемой силе индентор движется со скоростью, существенно превышающей значение, при котором контакт можно считать квазистатическим. При теоретическом описании процесса отрыва в условиях контролируемой силы необходимо учитывать вязкоупругость и влияние массы индентора на динамическую силу, наличие пружины и т. п. Учет этих эффектов не укладывается в стандартное описание разрушения контакта в условиях контролируемой силы в рамках классической теории JKR [11], поскольку теория не содержит информации о том, что происходит с контактом при достижении глубиной индентирования критического значения. В реальной жизни условия контролируемой силы встречаются очень часто. Самый простой пример - это когда мы стараемся разрушить клеевое соединение (проще говоря, что-либо оторвать) собственными руками. Однако из-за указанных выше проблем теоретического описания сегодня адгезионные контакты изучаются в основном в экспериментах с контролируемым перемещением.

\section{Заключение}

В настоящей работе экспериментально изучается влияние условий проведения эксперимента на адгезионную прочность контакта между резиновым образцом фиксированной толщины и сферическим стальным индентором, который по сравнению с резиной можно считать абсолютно жестким телом. Показано, что адгезионная прочность нарастает с увеличением времени продолжительности контакта и глубины индентирования. Проведенное исследование позволило выяснить, что при увеличении времени контакта и глубины индентирования контакт упрочняется согласно различным механизмам взаимодействия в контактной зоне. Показано совпадение результатов по индентированию в нормальном направлении, полученных непосредственно в эксперименте, рассчитанных теоретически и в компьютерном моделировании в рамках метода граничных элементов для двуслойных систем. Такое совпадение наблюдается для всех рассмотренных случаев. Поэтому работа может представлять интерес не только ввиду содержания в ней оригинальных экспериментальных результатов, а еще и как экспериментальное подтверждение указанных выше теоретических подходов расчета квазистатического адгезионного контакта. В работе были проведены два типа экспериментов - при условиях контролируемого перемещения и контролируемой силы. В обоих случаях указаны достоинства и недостатки получаемых данных в плане их теоретической интерпретации и практических приложений.

Дополнительные материалы к этой статье доступны в электронном виде (см. переводную версию).

\section{Финансирование работы}

Работа выполнена при финансовой поддержке Немецкого научно-исследовательского сообщества (DFG), проект РО 810-55-1.

\section{Конфликт интересов}

Авторы заявляют, что у них нет конфликта интересов. 


\section{Список литературы}

[1] Gorb S. Adhesion and friction in biological systems. Springer, 2012. $280 \mathrm{p}$.

[2] Ciavarella M., Joe J., Papangelo A., Barber J.R. // J. Roy. Soc. Interface. 2019. Vol. 16. N 151. 20180738. P. 1-22. https://doi.org/10.1098/rsif.2018.0738

[3] Schmitt P., Eberlein D., Ebert C., Tranitz M., Eitner I., Wirth H. // Energy Proced. 2013. Vol. 38. P. 380-386. https://doi.org/10.1016/j.egypro.2013.07.293

[4] Dmitriev A.I., Nikonov A.Y., Österle W. // Lubricants. 2016. Vol. 4. N 3.24. P. 1-12. https://doi.org/10.3390/lubricants4030024

[5] Carbone G., Pierro E., Gorb S.N. // Soft Matter. 2011. Vol. 7. N 12. P. 5545-5552. https://doi.org/10.1039/C0SM01482F

[6] Heepe L., Gorb S.N. // Ann. Rev. Mater. Res. 2014. Vol. 44. P. 173-203. https://doi.org/10.1146/annurev-matsci062910-100458

[7] Tang T., Hou X., Xiao Y., Su Y., Shi Y., Rao X. // Int. J. Adv. Robot. Syst. 2019. Vol. 16. N 1. P. 1-17. https://doi.org/10.1177/1729881418821578

[8] Deng W., Kesari H. // Sci. Rep. 2019. Vol. 9. 1639. P. 1-12. https://doi.org/10.1038/s41598-018-38212-z

[9] Ciavarella M., Papangelo A. // Phys. Mesomech. 2018. Vol. 21. N 1. P. 59-66. https://doi.org/10.1134/S1029959918010083

[10] Liu Z., Lu H., Zheng Y., Tao D., Meng Y., Tian Y. // Sci. Rep. 2018. Vol. 8. 6147. P. 1-8. https://doi.org/10.1038/s41598-01824587-6

[11] Johnson K.L., Kendall K., Roberts A.D. // Proc. Roy. Soc. Lond. A. 1971. Vol. 324. N 1558. P. 301-313. https://doi.org/10.1098/rspa.1971.0141

[12] Lyashenko I.A., Popov V.L// AIP Conf. Proc. 2019. Vol. 2167. N 1. 020201. P. 1-4. https://doi.org/10.1063/1.5132068

[13] Argatov I.I., Borodich F.M., Popov V.L. // J. Phys. D Appl. Phys. 2015. Vol. 49. N 4. 45307. P. 1-7. https://doi.org/10.1088/0022-3727/49/4/045307

[14] Hertz H.J // J. Reine Angew. Math. 1882. Vol. 92. P. 156-171. https://doi.org/10.1515/crll.1882.92.156

[15] Li Q., Pohrt R., Lyashenko I.A., Popov V.L. // P. I. Mech. Eng. J.-J. Eng. 2020. Vol. 234. N 1. P. 73-83. https://doi.org/10.1177/1350650119854250

[16] Maugis D. // J. Colloid Interf. Sci. 1992. Vol. 150. N 1. P. $243-$ 269. https://doi.org/10.1016/0021-9797(92)90285-T

[17] Stan G., Adams G.G. // Int. J. Solid. Struct. 2016. Vol. 87. N 1. P. 1-10. https://doi.org/10.1016/j.ijsolstr.2016.02.043

[18] Popov V.L., Pohrt R., Li Q. // Friction. 2017. Vol. 5. N 3. P. 308-325. https://doi.org/10.1007/s40544-017-0177-3

[19] Lyashenko I.A., Pohrt R. // Front. Mech. Eng. 2020. Vol. 6. 49. P. 1-10. https://doi.org/10.3389/fmech.2020.00049

[20] Li Q., Pohrt R., Popov V.L. // Front. Mech. Eng. 2019. Vol. 5. 7. P. 1-9. https://doi.org/10.3389/fmech.2019.00007

[21] Popov V.L. // AIP Conf. Proc. 2019. Vol. 2167. N 1. 020286. P. 1-6. https://doi.org/10.1063/1.5132153

[22] Pastewka L., Robbins M.O. // Proc. Natl. Acad. Sci. USA 2014. Vol. 111. N 9. P. 3298-3303. https://doi.org/10.1073/pnas.1320846111

[23] Dalvi S., Gujrati A., Khanal S.R., Pastewka L., Dhinojwala A., Jacobs T.D.B. // Proc. Natl. Acad. Sci. USA 2019. Vol. 116. N 51. P. 25484-25490. https://doi.org/10.1073/pnas.1913126116

[24] Johnson K.L. // Int. J. Solid. Struct. 1995. Vol. 32. N 3-4. P. 423-430. https://doi.org/10.1016/0020-7683(94)00111-9
[25] Persson B.N.J., Albohr O., Tartaglino U., Volokitin A.I., Tosatti E. // J. Phys. Cond. Matter. 2005. Vol. 17. N 1. P. R1-R62. https://doi.org/10.1088/0953-8984/17/1/R01

[26] Borodich F.M., Brousseau E., Clarke A., Pepelyshev A., Sánchez-López J.C. // Front. Mech. Eng. 2019. Vol. 5.24. P. 1-13. https://doi.org/10.3389/fmech.2019.00024

[27] Style R.W., Hyland C., Boltyanskiy R., Wettlaufer J.S., Dufresne E.R. // Nat. Commun. 2013. Vol. 4. 2728. P. 1-6. https://doi.org/10.1038/ncomms3728

[28] Salez T., Benzaquen M., Raphaël É. // Soft Matter. 2013. Vol. 9. P. 10699-10704. https://doi.org/10.1039/C3SM51780B

[29] Popov V.L., Heß M. Method of dimensionality reduction in contact mechanics and friction. Berlin: Springer, 2015. 265 p. https://doi.org/10.1007/978-3-642-53876-6

[30] Argatov I. // Fact. Univ. Ser. Mech. Eng. 2019. Vol. 17. N 2. P. 181-190. https://doi.org/10.22190/FUME190330024A

[31] Jin F., Guo X. // Int. J. Solid. Struct. 2013. Vol. 50. N 20-21. P. 3375-3386. https://doi.org/10.1016/j.ijsolstr.2013.06.007

[32] Jacobs T.D.B., Ryan K.E., Keating P.L., Grierson D.S., Lefever J.A., Turner K.T., Harrison J.A., Carpick R.W. // Tribol. Lett. 2013. Vol. 50. N 1. P. 81-93. https://doi.org/10.1007/s11249-012-0097-3

[33] Scheibert J., Sahli M., Peyrard M. // Front. Mech. Eng. 2020. Vol. 6. 18. P. 1-9. https://doi.org/10.3389/fmech.2020.00018

[34] Tolpekina T.V., Persson B.N.J. // Lubricants. 2019. Vol. 7. N 3.20. P. 1-25. https://doi.org/10.3390/lubricants7030020

[35] Li Q., Kim K.S. // Acta Mech. Solida Sin. 2009. Vol. 22. N 5. P. 377-390. https://doi.org/10.1016/S0894-9166(09)60288-3

[36] Sahli R., Pallares G., Papangelo A., Ciavarella M., Ducottet C., Ponthus N., Scheibert J. // Phys. Rev. Lett. 2019. Vol. 122. N 21. 214301. P. 1-6. https://doi.org/10.1103/PhysRevLett.122.214301 